ISSN: $2317-8957$

Volume 5, Number 2, Dec. 2017

\title{
FINANCIAMENTO DO SUS: DISTRIBUIÇÃO, GESTÃO E ALOCAÇÃO DE RECURSOS NA ATENÇÃO BÁSICA
}

\author{
LIZ RODRIGUES DE SOUZA, ANA LÚCIA TORRES DEVEZAS SOUZA
}

1 UniFOA - Centro Universitário de Volta Redonda

\begin{abstract}
RESUMO
O presente estudo trata de uma pesquisa documental com objetivo exploratório de natureza aplicada e abordagem quali-quantitativa acerca do financiamento do Sistema Único de Saúde (SUS) e a gestão de recursos destinados à atenção básica. Entende-se que os princípios da eficácia e da eficiência não estão atrelados somente aos recursos humanos. Há série de fatores que influenciam a qualidade de ações e serviços prestados à comunidade. Tal assertiva impacta diretamente na análise de qualidade do Programa de Educação pelo Trabalho para a Saúde Pet GraduaSUS. Esse programa consiste em uma parceria entre o Centro Universitário de Volta Redonda - UniFOA e as Secretarias Municipais de Saúde de Volta Redonda e Pinheiral com o objetivo de desenvolver uma ação intersetorial que visa o fortalecimento de áreas estratégicas para o - SUS, por meio da integração ensino-serviçocomunidade. O UniFOA em parceria com desde o início de suas atividades em maio de 2016, vem inserindo discentes da graduação de Enfermagem na rede de atenção básica dos referidos municípios. Esses estudantes têm a atribuição de observar e desenvolver atividades de assistência e gestão. No entanto, observa-se durante a execução das atividades, dificuldades no planejamento, implementação e execução de algumas ações, seja por falta de recursos materiais, financeiros e humanos. O estudo documental buscou identificar o financiamento da atenção básica no município de Volta Redonda através da aplicação da verba repassada pelo fundo nacional de saúde e o impacto da uti-lização destas verbas para melhorar os indicadores de saúde. Após análise de dados, pode-se observar que o aumento progressivo do repasse nos últimos anos não resultou em melhoria na cobertura da assistência, o que torna a necessidade de um planejamento adequado e a utilização dos repasses como ponto de partida para o cumprimento dos princípios do Sistema Único de Saúde, com cobertura populacional de excelência.
\end{abstract}

Palavras-chave: Atenção básica, gestão, recursos financeiros, sistema único de saúde.

\begin{abstract}
The present study is about a documental research with an exploratory objective of an applied nature and quantitative and qualitative approach on the sector of the Unified Health System ( SUS) and the management of resources for basic care. It is understood that the principles of efficiency and the effectiveness are not tied only to human resources. There are a series of factors that influence the quality of actions and services provided to the community. This assertion directly impacts on the quality analysis of the Education for Work Program for Health Pet GraduaSUS. This program consists of an intersectoral action at strengthening strategic areas for the SUS, through the integration of teaching-service-community. The UNIFOA in partnership with the Municipal Health Secretaries of Volta Redonda and Pinheiral since the beginning of its activities on May 2016, has been inserting nursing students in the basic health care network of referred counties. These students are assigned to observe and develop activities of assistance and management. However, it is worth mentioning that during the execution of the activities, difficulties were observed in the planning, implementation and execution of some of actions, either for lack of material, financial and human resources. The documental study search to identify the financing of basic care in the county of Volta Redonda through the application of the funds provided by the national health fund and the impact of the use of these funds to improve the health indicators of the city. After data analysis, it can be observe that the progressive increase of the pass-through in last years didn't result in an improvement in the coverage of care, which makes the need for adequate planning of the use of transfers starting point for compliance with the principles of the Unified Health System, with a population coverage of excellence.
\end{abstract}

Keywords: basic healthcare, management, financial resources, sus.

\section{INTRODUÇÃO}

A lei $\mathrm{n}^{\circ} 8.080$ de 19 de setembro de 1990 defende a saúde como um direito fundamental do ser humano e afirma ser dever do Estado prover as condições indispensáveis ao seu pleno exercício. Para isso, deve garantir formulação e execução de políticas econômicas e soci-ais que visem a redução de riscos de doenças e de outros agravos, além do estabelecimento de condições que assegurem acesso universal e igualitário às ações e aos serviços para a sua pro-moção, proteção e recuperação (Brasil, 1990). De acordo com a Constituição Federal 
de 1988, art. 30, inciso VII, compete aos Municípios prestar, com a cooperação técnica e financeira da União e do Estado, serviços de atendimento à saúde da população. Nela também, o art 35, inciso III afirma que o Estado não deverá intervir em seus Municípios, nem a União nos Municípios localizados em Território Federal, exceto quando não tiver sido aplicado o mínimo exigido da receita municipal na manutenção e desenvolvimento do ensino e nas ações e serviços públicos de saúde (Brasil, 1988).

A Portaria GM/MS n. 204, de 29 de janeiro de 2007, regulamenta o financiamento e a transferência dos recursos federais para as ações e serviços de saúde, ambos com monitoramento e controle. Os recursos federais destinados às ações e serviços de saúde são então organizados e transferidos na forma de Blocos de Financiamento. Os blocos são: Atenção Básica, Atenção de Média e Alta Complexidade Ambulatorial e Hospitalar, Vigilância em Saúde, Assistência Farmacêutica, Gestão do SUS e Investimentos na Rede de Serviços de Saúde.

Esses blocos são constituídos por componentes, de acordo com as especificidades de suas ações e os serviços de saúde pactuados. Os recursos federais que compõem cada bloco de financiamento são transferidos aos estados, Distrito Federal e municípios, fundo a fundo, em conta única e específica para cada bloco de financiamento, observados os atos normativos específicos.

A Lei Complementar $n^{\circ} 141$ dispõe sobre os valores mínimos a serem aplicados anualmente pela União, Estados, Distrito Federal e Municípios em ações e serviços públicos de saúde além de estabelecer os critérios de rateio dos recursos de transferências para a saúde e as normas de fiscalização, avaliação e controle das despesas com saúde nas 3 (três) esferas de governo.

$\mathrm{O}$ artigo $5^{\circ}$ da lei complementar $\mathrm{n}^{\circ} 141$ afirma que a União deve aplicar anualmente o montante correspondente ao valor empenhado no exercício financeiro anterior, acrescido de, no mínimo, o percentual correspondente à variação nominal do Produto Interno Bruto (PIB) ocorrida no ano anterior ao da lei orçamentária anual. No que diz respeito aos estados e Distrito Federal, o artigo $6^{\circ}$ afirma que deverá ser aplicado anualmente o valor referente a, no mínimo, 12\% (doze por cento) da arrecadação dos impostos, enquanto para os municípios, o valor anual aplicado em ações e serviços públicos de saúde é, no mínimo, 15\% (quinze por cento) da arrecadação dos impostos. Solha (2014), afirma que os recursos são provenientes dos impostos pagos pela população, de forma direta, como o IPVA Imposto sobre a Propriedade de Veículos Automotores, pago direto ao estado ou o ISS Imposto sobre Serviços, pago ao município, e indiretamente, como o ICMS - Imposto sobre Circulação de Mercadorias, que pagamos ao comprar alguma mercadoria. Outra fonte de recursos provem das contribuições sociais de empregadores e empregados, como o Fundo de Garantia por Tempo de Serviço - FGTS e contribuições previdenciárias para o Instituto Nacional de Seguridade Social - INSS.

O Conselho Nacional de Saúde emitiu a Resolução $n^{\circ} 322$, de 8 de maio de 2003, que apresenta o que e quais são as despesas com ações e serviços públicos de saúde, buscando orientar e direcionar para o uso correto das verbas. Entre as despesas com ações e serviços públicos de saúde definidas pela resolução, destacam-se a vigilância epidemiológica e controle de doenças, vigilância sanitária, vigilância nutricional, controle de deficiências nutricionais, orientação alimentar, e a segurança alimentar promovida no âmbito do SUS, educação para a saúde, saúde do trabalhador, assistência à saúde em todos os níveis de complexidade, assistência farmacêutica, atenção à saúde dos povos indígenas, capacitação de recursos humanos do SUS, pesquisa e desenvolvimento científico e tecnológico em saúde, promovidos por entidades do SUS, produção, aquisição e distribuição de insumos setoriais específicos, tais como medicamentos, imunobiológicos, sangue e hemoderivados, e equipamentos, saneamento básico e do meio ambiente, desde que associado diretamente ao controle de 
vetores, a ações próprias de pequenas comunidades ou em nível domiciliar, ou aos Distritos Sanitários Especiais Indígenas (DSEI), serviços de saúde penitenciários, desde que firmado Termo de Cooperação especifico entre os órgãos de saúde e os órgãos responsáveis pela prestação dos referidos serviços, atenção especial aos portadores de deficiência, ações administrativas realizadas pelos órgãos de saúde no âmbito do SUS e indispensáveis para a execução das ações indicadas nos itens anteriores (Brasil, 2003).

O Piso de Atenção Básica (PAB), foi criado em 1997 e implantado em 1998, e é um mecanismo de financiamento no Sistema Único de Saúde com uma nova sistemática, onde os fundos municipais de saúde recebem diretamente do Fundo Nacional de Saúde um montante per capita previamente fixado e a administração local se responsabiliza pela atenção básica à saúde da população de seu território. Neste contexto, a prefeitura deixa de ser tratada como prestadora de serviços e o secretário de saúde passa a exercer papel de gestor do SUS, elaborando planos e ações em saúde de acordo com a realidade local e visando um sistema de saúde mais equitativo e eficiente (Brasil, 2001).

"A atenção básica caracteriza-se por um conjunto de ações de saúde, no âmbito individual e coletivo, que abrange a promoção e a proteção da saúde, a prevenção de agravos, o diagnóstico, o tratamento, a reabilitação, a redução de danos e a manutenção da saúde com o objetivo de desenvolver uma atenção integral que impacte na situação de saúde e autonomia das pessoas e nos determinantes e condicionantes de saúde das coletividades." (Brasil, 2012).

Essa atenção é desenvolvida por meio de práticas de cuidado e gestão, através do trabalho de equipe, direcionadas a populações de territórios definidos, pelas quais assume a responsabilidade sanitária, considerando a dinâmica existente nesses territórios.

No Brasil, a Atenção Básica é desenvolvida com descentralização e capilaridade, o que contribui para que a atenção à população ocorra em locais mais próximos da vida das pessoas, o que consequentemente a faz o con- tato preferencial dos usuários e a principal porta de entrada na RAS - Rede de Atenção à Saúde, além de ser um importante centro de comunicação com toda a rede (Brasil, 2012).

Dentre as funções que a Atenção Básica deve cumprir, de acordo com a Política Nacional de Atenção Básica, destacam-se: ser a modalidade de atenção e de serviço de saúde com o mais elevado grau de descentralização e capilaridade, cuja participação no cuidado se faz sempre necessária; identificar riscos, necessidades e demandas de saúde, utilizando e articulando diferentes tecnologias de cuidado individual e coletivo, por meio de uma clínica ampliada capaz de construir vínculos positivos e intervenções clínica e sanitária efetivas, na perspectiva de ampliação dos graus de autonomia dos indivíduos e grupos sociais; elaborar, acompanhar e gerir projetos terapêuticos singulares, bem como acompanhar e organizar o fluxo dos usuários entre os pontos de atenção das RAS; reconhecer as necessidades de saúde da população sob sua responsabilidade, organizando-as em relação aos outros pontos de atenção, contribuindo para que a programação dos serviços de saúde parta das necessidades de saúde dos usuários (Brasil, 2012).

O PET-Saúde GraduaSUS, Programa de Educação pelo Trabalho para a Saúde é regulamentado pela Portaria Interministerial $n^{\circ}$ 421, de 03 de março de 2010. Ele busca ações intersetoriais direcionadas para o fortalecimento de áreas estratégicas para o Sistema Único de Saúde - SUS, a partir da educação pelo trabalho, envolvendo tutores, preceptores e estudantes de graduação da área da saúde através da integração ensino-serviço-comunidade.

Nas cidades de Volta Redonda e Pinheiral, o programa foi implantado com as parcerias das Secretarias Municipais de Saúde (SMS) e o Centro Universitário de Volta Redonda - UniFOA. Os tutores e os alunos são do Centro Universitário de Volta Redonda, e os preceptores são funcionários da área da Saúde das prefeituras de Volta Redonda e Pinheiral.

Ao participar do programa, pôde-se observar dificuldades na implementação e 
execução de algumas ações pelos profissionais de saúde da rede, seja por deficiência de recursos humanos, financeiros e materiais.

Diante do que foi exposto, surgem as seguintes perguntas: Quanto de recurso que a gestão recebe é repassado para a Atenção Básica? Os recursos hoje disponíveis estão sendo suficientes para a execução de procedimentos e ações na atenção básica do Município de Volta Redonda? Será que só o aumento do repasse é necessário para melhorar a cobertura da assistência?

Sendo assim, o seguinte trabalho buscou identificar o financiamento da atenção básica no município de Volta Redonda através da aplicação da verba repassada pelo fundo nacional de saúde e o impacto da utilização destas verbas para melhorar os indicadores de saúde do município.

\section{METODOLOGIA}

Pesquisa de natureza aplicada, visto que, segundo Gerhardt e Silveira (2009), essa linha objetiva gerar conhecimentos para aplicação prática dirigidos à solução de problemas específicos, além de envolver verdades e interesses locais. Possui uma abordagem quantitativa e qualitativa, pois procura traduzir numericamente informações para serem classificadas e analisadas, mas também procura descrever informações obtidas e não quantificáveis, pressupondo interpretação de fenômenos.

Uma pesquisa documental com objetivo exploratório, pois se baseia em levantamento bibliográfico e análise profunda de manuais, legislações federais, estaduais e municipais acerca do financiamento e gestão de recursos para o Sistema Único de Saúde além de instrumentos de gestão e plano municipal de saúde da cidade, com objetivo de verificar o direcionamento dos recursos da Atenção Básica na gestão Municipal.

Os dados financeiros a serem analisados foram obtidos e extraídos do site do Fundo Nacional de Saúde, por meio da consulta de pagamento detalhada. Nela os blocos Atenção Básica e Investimentos na Rede de Serviços em Saúde foram selecionados e foram avaliados os últimoscincoanos:2012,2013,2014,2015e2016.

Os dados de cobertura populacional da Atenção Básica e da Saúde Bucal foram solicitados e obtidos na Secretaria de Saúde do Município de Volta Redonda, no setor de Epidemiologia, pois não foram encontrados dados atualizados em outros sites.

Realizou-se uma pesquisa sobre como o financiamento e repasses devem ser direcionados à Atenção Básica, um levantamento dos valores repassados nos últimos cinco anos e uma comparação com os índices de cobertura populacional do município nos mesmos anos, a fim de responder às perguntas e atingir os objetivos do trabalho.

\section{DISCUSSÃO}

A Portaria $n^{\circ} 204$, de 29 de janeiro de 2007, regulamentando o financiamento e a transferência dos recursos federais para as ações e os serviços de saúde, na forma de blocos de financiamento, com o respectivo monitoramento e controle afirma ser de responsabilidade das três esferas de gestão do Sistema Único de Saúde - SUS - o financiamento das ações e serviços de saúde, embasado na Constituição Federal e na Lei Orgânica da Saúde e define cinco blocos de financiamento: Atenção Básica, Atenção de Média e Alta Complexidade Ambulatorial e Hospitalar, Vigilância em Saúde, Assistência Farmacêutica e Gestão do SUS. Além desses blocos, a portaria apresenta um sexto bloco, Investimentos na Rede de Serviços de Saúde, em que os recursos destinados a ele serão destinados exclusivamente às despesas de capital. A Política Nacional de Atenção Básica - PNAB (Brasil, 2012) declara que o financiamento da atenção básica deve ser tripartite e que os recursos financeiros destinados às ações da atenção básica compõem o Bloco de Financiamento de Atenção Básica e parte do Bloco de Financiamento de Investimento. Esses recursos deverão ser 
distribuídos, alocados e utilizados de forma a financiar as ações de Atenção Básica descritas na Relação Nacional de Ações e Serviços de Saúde - RENASES - e nos planos de saúde do município.

No Bloco da Atenção Básica, o mecanismo antes realizado para financiamento do Sistema Único de Saúde (SUS) possuía como lógica o pagamento pela produção, porém este começou a sofrer inúmeras críticas por destacar o financiamento da doença à frente da atenção integral à saúde, além de privilegiar as localidades que já possuíam estrutura e serviços. Pensando nisso, foi implantado o Piso de Atenção Básica (PAB), que busca uma nova sistemática de financiamento, onde os municípios recebem verba diretamente de um repasse fundo a fundo, vindo do Fundo Nacional de Saúde, através de um montante calculado de acordo com o número de habitantes residentes no município e após recebido, a responsabilidade pela atenção básica passa ser estritamente da administração local (BRASIL, 2001). As prefeituras passam a necessitar de gestores de saúde, para que esses valores sejam melhor distribuídos e alocados, deixando de ser apenas prestadoras de serviços. O objetivo principal dessa mudança é um sistema de saúde mais eficiente e buscando sempre equidade, se baseando em promoção de saúde e prevenção de doenças, trazendo ganhos e qualidade de vida à população.

O Piso da Atenção Básica (PAB) é composto por uma parte fixa e uma parte variável. A parte fixa é destinada à assistência básica, enquanto a variável é destinada especificamente ao desenvolvimento de ações da atenção básica, como Vigilâncias Sanitária, Epidemiológica e Ambiental, Programas de Agentes Comunitários, Assistência farmacêutica, etc.

Em relação à utilização dos repasses, cabe salientar que os valores repassados para os municípios devem ser direcionados apenas para responsabilidades definidas para a gestão da Atenção Básica, coerentes com o que direciona o Plano Municipal de Saúde de cada um.

Os registros dos serviços de saúde prestados à população, a gestão municipal e à análise de dados estatísticos são feitas através de uma avaliação por um mecanismo chamado Sistema de Informação em Saúde. Dentre os principais, pode-se destacar o Sistema de Informação Sobre Agravos de Notificação (SINAN), Sistema de Informação sobre Mortalidade (SIM), Sistema de Informação Sobre Nascidos Vivos (SINASC) e Sistema de Vigilância Alimentar e Nutricional (SISVAN). Caso não ocorra alimentação desses sistemas por dois meses consecutivos pelos municípios, a transferência de recursos do PAB poderá ser suspensa.

No Bloco de Investimento, de acordo com a Portaria $n^{\circ} 837$, de 23 de abril de 2009, que altera e acrescenta dispositivos à Portaria $n^{\circ}$ 204/GM, de 29 de janeiro de 2007, para inserir o Bloco de Investimentos na Rede de Serviços de Saúde na composição dos blocos de financiamento relativos à transferência de recursos federais para as ações e os serviços de saúde no âmbito do Sistema Único de Saúde - SUS, os recursos financeiros a serem transferidos por meio do Bloco de Investimentos na Rede de Serviços de Saúde destinar-se-ão exclusivamente às despesas de capital.Esse Bloco é composto por recursos financeiros que serão transferidos, mediante apresentação de projeto, encaminhado pelo ente federativo interessado, ao Ministério da Saúde através de repasse regular e automático do Fundo Nacional de Saúde para os Fundos de Saúde Estaduais, Municipais e do Distrito Federal.Após busca, coleta de dados e análise no endereço da web do Fundo Nacional de Saúde, pôde-se obter os investimentos do Bloco da Atenção Básica através do PAB e do Bloco de Investimentos na Rede de Serviços de Saúde no Município de Volta Redonda nos últimos cinco anos. As tabelas a seguir apresentam seus valores.Como pode-se observar, o valor total do PAB Fixo cresceu nos últimos cinco anos, passando de 5.493.180,20 em 2012 para 7.820.732,32 em 2016. Porém nesse acréscimo também estão incluídos outros valores que se enquadram no Piso Fixo, como repasses para reformas, incrementos, informatização e telessaúde. 
Tabela 1: PAB Fixo do Município de Volta Redonda dos últimos cinco anos.

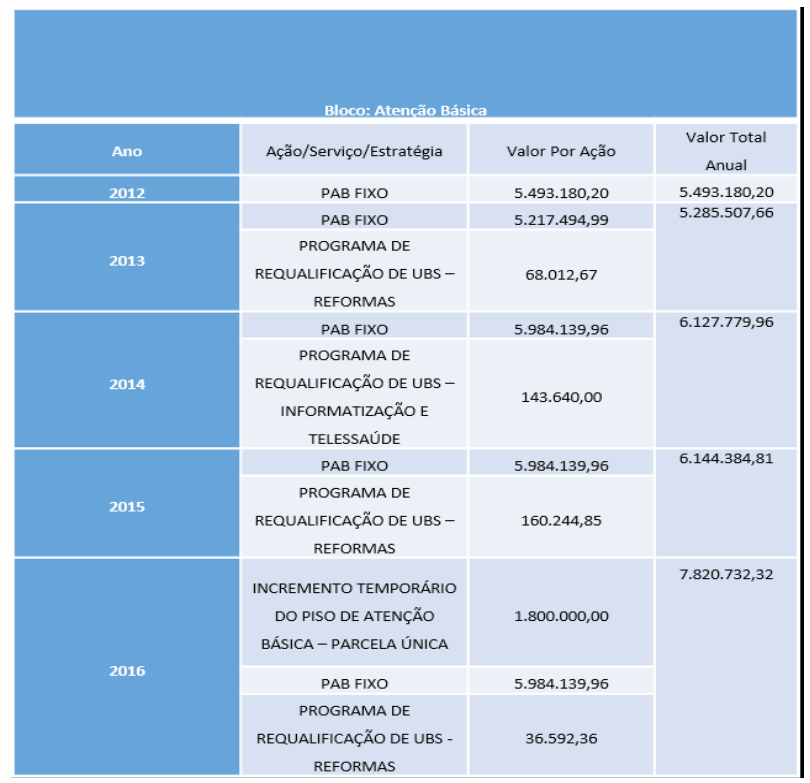

Tabela 2: PAB Variável do Município de Volta Redonda dos últimos cinco anos

\begin{tabular}{|c|c|c|c|}
\hline & Bloco: Atenção Básica & & \\
\hline Ano & Ação/Serviço/Estratégia & Valor por ação & Valor Total Anual \\
\hline \multirow{2}{*}{2012} & PAB Variável & & \multirow{2}{*}{$14.315 .412,72$} \\
\hline & SAÚDE BUCAL - SB & $1.092 .670,00$ & \\
\hline \multirow[t]{2}{*}{2013} & PAB Variável & & \multirow[t]{2}{*}{$13.767 .862,27$} \\
\hline & SAÚDE BUCAL - SB & $929.900,00$ & \\
\hline \multirow[t]{2}{*}{2014} & PAB Variável & & \multirow[t]{2}{*}{$12.196 .154,03$} \\
\hline & SAÚDE BUCAL - SB & $948.470,00$ & \\
\hline \multirow[t]{2}{*}{2015} & PAB Variável & & \multirow[t]{2}{*}{$12.946 .695,25$} \\
\hline & SAÚDE BUCAL - SB & $1.155 .940,00$ & \\
\hline \multirow[t]{2}{*}{2016} & PAB Variável & & \multirow[t]{2}{*}{$12.697 .578,97$} \\
\hline & SAÚDE BUCAL - SB & $1.219 .110,00$ & \\
\hline
\end{tabular}

Quanto ao PAB Variável, o valor total repassado anualmente reduziu, passando de 14.315.412,72 no ano de 2012 para 12.697.578,97 no ano de 2016. A tabela 2 apresenta o valor total anual de repasses de PAB Variável entre os anos de 2012 e 2016 e o valor anual do PAB Variável destinado à ação de Saúde Bucal, que foi destacado para discussão do trabalho.
Tabela 3: Investimentos na Rede de Serviços de Saúde dos últimos cinco anos

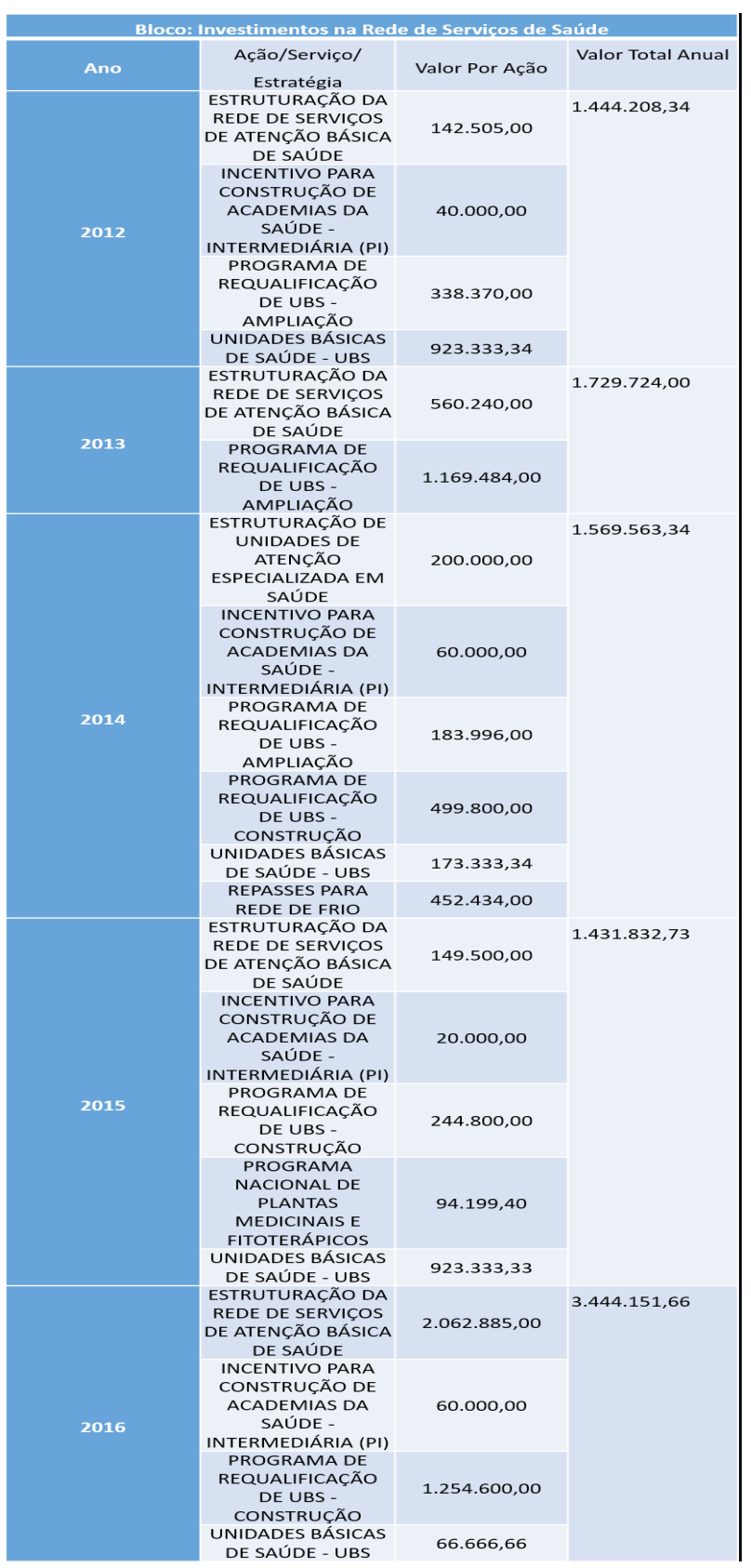

No Bloco de Investimento na Rede de Serviços em Saúde, o valor de repasse para a Atenção Básica teve um aumento significativo no ano de 2016, quando comparado às oscilações de repasses dos anos anteriores. O investimento passou de 1.444.208,34 no ano de 2012 para 3.444.151,66 no ano de 2016. Vale observar que neste bloco destacam-se projetos atrelados à Atenção Básica como a Academia da Saúde, Rede de Frios e o Programa Nacional de Plantas Medicinais e Fitoterápicos. 
A Política Nacional de Atenção Básica PNAB (Brasil, 2012), assegura que o Plano Municipal de Saúde deve especificar a proposta de organização da atenção básica e explicitar como serão utilizados os recursos recebidos através do Fundo Nacional de Saúde (FNS). A Relação Nacional de Ações e Serviços de Saúde - RENASES define como ações da Atenção Primária:

“(...) promoção à saúde; prevenção de agravos; vigilância à saúde; tratamento, acompanhamento, redução de danos e reabilitação, com ênfase nas necessidades e problemas de saúde de maior frequência e relevância em seu território/contexto, observando critérios de riscos e vulnerabilidades; acolhimento e atenção à demanda espontânea incluindo as urgências e emergências nas unidades básicas de saúde; atenção e cuidado continuado/programado tanto nas unidades de saúde como em domicílio (quando for necessário); indicação, prescrição e realização de procedimentos terapêuticos e diagnósticos no âmbito da Atenção Primária; atividades de atenção individual e coletivas (ex.: Familiar, Comunitária); atividades de vigilância em saúde; atenção a todos os cidadãos sob sua responsabilidade, independente dos ciclos de vida, gênero ou problema de saúde apresentados; ações de Atenção Domiciliar; atenção à Saúde Bucal; atenção através de ações e serviços pertinentes às Práticas Integrativas e Complementares; Vigilância Nutricional e Alimentar e Atenção Nutricional; coordenação do cuidado, incluindo o acesso a ações e serviços fora do âmbito da Atenção Primária.” (BRASIL, 2012)

Como o período analisado foram os últimos cinco anos, ao avaliar o Plano Municipal de Saúde do Município de Volta Redonda, encontram-se duas versões, a que compreende o período de 2011 a 2013 e outra de 2014 a 2017. Os Planos Municipais possuem informações como dados epidemiológicos do município, dados vitais e ações estratégicas. Para este estudo a Atenção Básica foi explorada dentro de Ações Estratégicas a Alocação de Recursos e dentro de Gestão das Ações em Saúde.
No Plano Municipal de Saúde 2011/2013, consta que a população estava dividida em territórios, denominados Distritos Sanitários, Norte e Sul, que possuíam a missão de assegurar a toda população eficiência de serviços organizados com base em ações oferecidas dentro de uma base territorial. O plano mostrou que entre 2008 e 2010 a cobertura populacional da Estratégia de Saúde da Família ampliou, devido a esta forma de trabalho descentralizada, com práticas gerenciais e sanitárias democráticas e participativas.

Para os anos entre 2011 e 2013, o Plano apresentou, entre as principais ações o planejamento de ampliação da rede de saúde da Atenção Primária, com 06 novas Unidades de Saúde e implantação do Programa de Agentes Comunitários de Saúde em um dos bairros, ProjetosPilotosemregiõesdealtavulnerabilidade visando a ampliação do acesso e da incorporação tecnológica nesses territórios, ampliação e promoção de melhorias e manutenção em 10 Unidades de Saúde da Família, qualificação da assistência farmacêutica como estratégia de atenção à Saúde com ênfase para a Atenção Básica, desenvolvimento de linhas de cuidado com prioridade para hipertensão e diabetes, qualificação de ações de Vigilância em Saúde, capacitação de profissionais, implantação da rede de informática nas Unidades de Saúde e implantação de sistema de gerenciamento de insumos e medicamentos. No segundo Plano Municipal de Saúde, que compreende os anos de 2014 a 2017, a lógica dos Distritos Sanitários se manteve, para uma melhor gestão e assistência das Unidades Básicas. Para esse período o plano apresentou, entre as principais ações o planejamento e ampliação da rede de saúde, com construção e reforma de 15 Unidades Básicas (sendo 14 de Saúde da Família e 01 Centro Odontológico) e 01 Academia da Saúde, Projetos Pilotos em regiões de alta vulnerabilidade visando a ampliação do acesso e da incorporação tecnológica nesses territórios, promoção de melhorias em 27 Unidades Básicas, sendo 02 Odontológicas, qualificação da assistência farmacêutica como 
27 Unidades Básicas, sendo 02 Odontológicas, qualificação da assistência farmacêutica como estratégia de atenção à Saúde com ênfase para a Atenção Básica, orientação das ações de saúde na $A B$ pelos princípios da universalidade, da acessibilidade, do vínculo, da continuidade do cuidado, da integralidade da atenção, da responsabilização, da humanização, da equidade e da participação social, qualificação de ações de Vigilância em Saúde, abastecimento de insumos em toda Rede e incorporação tecnológica.

Foram analisados os resultados de dois indicadores diretamente relacionados à Atenção Básica nos últimos cinco anos: Cobertura populacional estimada pelas equipes de Atenção Básica e Cobertura populacional estimada de saúde bucal na Atenção Básica. Esses dados foram retirados através do programa de pactuação de metas do Governo do Estado do Rio de Janeiro.

Tabela 4: Cobertura populacional estimada pelas equipes de

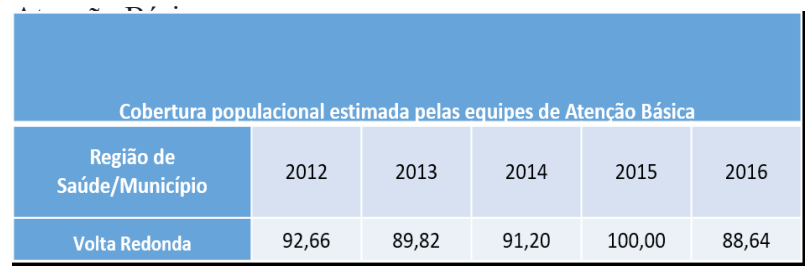

Tabela 5: Cobertura populacional estimada de saúde bucal na

\begin{tabular}{|c|c|c|c|c|c|}
\hline $\begin{array}{c}\text { Regiảo de } \\
\text { Saúde/Município }\end{array}$ & 2012 & 2013 & 2014 & 2015 & 2016 \\
\hline Volta Redonda & 82,58 & 82,21 & 81,62 & 80,19 & 77,80 \\
\hline
\end{tabular}

Com base nos dados já apresentados, podemos fazer uma comparação com o valor de repasse destinado a Atenção Básica e sua cobertura populacional nos últimos cinco anos. Importante destacar que, para confecção do primeiro gráfico, foi separado o valor total anual de recursos, que representa o somatório do $\mathrm{PAB}$ Fixo, PAB Variável e bloco de Investimentos na Rede de Serviços de Saúde. No segundo gráfico, o valor representado é apenas o que se refere à Saúde Bucal, no PAB Variável.

No primeiro gráfico (Fig 1) vemos que no ano de 2012 a cobertura populacional ficou em $92,66 \%$, enquanto o valor de repasse total para atenção básica foi de 21.252.801,26.
No ano de 2013, o valor da cobertura diminuiu um percentual de 2,84, passando para $89,82 \%$ juntamente com o valor de repasse que diminuiu um percentual de 2,21, passando para 20.783.093,93. No ano de 2014 mesmo com redução no valor anual do repasse de $4,31 \%$, a cobertura populacional aumentou $1,38 \%$. Os anos de 2015 e 2016 tiveram um aumento progressivo de repasse, $3,06 \%$ e $14,3 \%$ respectivamente, porém, o valor de $100 \%$ de cobertura populacional conquistado em 2015 foi reduzido para $88,64 \%$ no ano de 2016 , o que indica uma queda de percentual de 11,36.

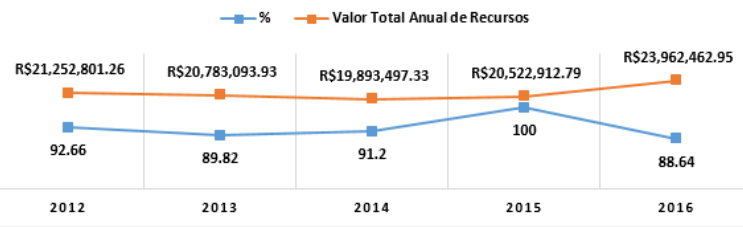

Figura 1: Cobertura populacional estimada pelas equipes de Atenção Básica

No segundo gráfico (Fig 2), o valor de repasse utilizado para análise foi o destinado a Saúde Bucal, presente no PAB Variável, descrito e especificado nas tabelas acima. Observamos que do ano de 2012 para 2013, a cobertura populacional sofreu uma queda de $0,37 \%$, enquanto o valor do repasse reduziu $14,9 \%$. No ano de 2014 , o repasse subiu $1,96 \%$ enquanto a cobertura populacional continuou sofrendo reduções, dessa vez de $0,59 \%$. Em 2015 os investimentos e repasses continuaram subindo, apresentando um aumento de $18 \%$, enquanto a cobertura populacional permaneceu em queda com $1,43 \%$ quando comparada ao ano anterior. 2016 não foi diferente, e apresentou um aumento de investimentos de 5,2\% quando comparado ao ano anterior e uma queda na cobertura populacional de 2,39\%.

Assim, pode-se perceber que a cobertura populacional reduziu gradativamente no decorrer dos cinco anos, passando de $82,58 \%$ no ano de 2012 para $77,8 \%$ no ano de 2016 . Porém, o valor dos repasses, que teve uma queda significativa no ano de 2013, passando de 1.092.670,00 em 2012 para 929.900,00 


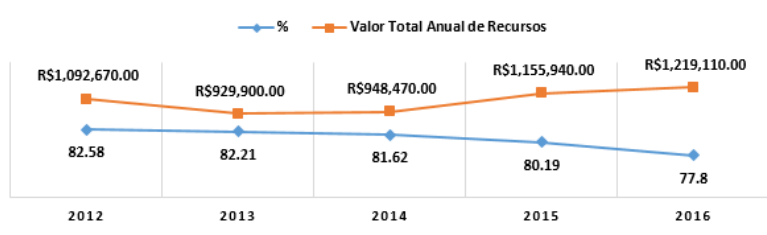

Figura 2: Cobertura populacional estimada de saúde bucal nas equipes de Atenção Básica

\section{CONCLUSÃO}

Com base nos dados, vemos que de maneira geral, os repasses feitos fundo a fundo para a Atenção Básica sofrem muitas variações quando comparados anualmente. Seus valores variam de acordo com número populacional, projetos apresentados para financiamento, alimentação de Sistemas de Informação de Saúde e ações desenvolvidas, além de sofrer variações de acordo com o valor do Produto Interno Bruto do país.

Outro aspecto relevante é que, mesmo com as variações dos valores de repasses, podese verificar que em números totais, somando os repasses dos dois blocos, o investimento tem aumentado progressivamente a partir do ano de 2014. Porém, mesmo com o aumento progressivo de investimentos, observamos uma queda na Cobertura populacional estimada pelas equipes de Atenção Básica do ano de 2015 para 2016, e uma queda contínua na Cobertura populacional estimada de saúde bucal na Atenção Básica desde o ano de 2012.

Assim, infere-se que o aumento progressivo dos repasses nos últimos anos não resultou em melhoria na cobertura da assistência na Atenção Básica, indicando a necessidade de um planejamento adequado da utilização dos repasses, através de adequação das atividades, tendo os indicadores de saúde como balizadores para nortear as ações à serem realizadas.

Acredita-se que exista uma interferência significativa dos interesses políticospartidários, que acabam interferindo não só no planejamento, como também na elaboração de propostas e leis, voltadas para interesses privados em detrimento do bem público. Por estar previsto que o direcionamento dos recursos, bem como o planejamento das atividades a serem desenvolvidas cabem à gestão de cada município, torna-se clara também a necessidade de gestores capacitados e preocupados com o bem público, para uma busca constante do cumprimento dos princípios e diretrizes do Sistema Único de Saúde.

Através deste estudo pode-se perceber que paralelamente ao aumento no repasse, ocorreram mudanças nas pactuações dos indicadores de saúde; que por vezes em situações pontuais apresentaram melhora, mas, que não foi possível a sustentabilidade do percentual atingido. Como sugestão para próximas pesquisas, fica o questionamento: Porque não foi possível esta sustentabilidade? Através de reformulação da forma de gestão do Sistema Único de Saúde, será possível atender o que é publicado na constituição: "Saúde dever do Estado e Direito do Cidadão"?

\section{REFERÊNCIAS BIBLIOGRÁFICAS}

BRASIL, Conselho Nacional de Saúde. Resolução no 322, de 08 de maio de 2003. Aprovação de diretrizes acerca da aplicação da Emenda Constitucional $n^{\circ} 29$, de 13 de setembro de 2000. Brasília, 2003.

BRASIL. Conselho Nacional de Secretários de Saúde. Para entender a gestão do SUS / Conselho Nacional de Secretários de Saúde. - Brasília : CONASS, 2003.

BRASIL. Constituição (1988). Constituição da República Federativa do Brasil. Brasília, DF: Senado Federal: Centro Gráfico, 1988. 292 p.

BRASIL. Lei complementar $n^{\circ} 141$, de 13 de janeiro de 2012. Regulamenta o § 3o do art. 198 da Constituição Federal para dispor sobre os valores mínimos a serem aplicados anualmente pela União, Estados, Distrito Federal e $\mathrm{Mu}-$ nicípios em ações e serviços públicos de saúde; estabelece os critérios de rateio dos recursos de transferências para a saúde e as normas de 
fiscalização, avaliação e controle das despesas com saúde nas 3 (três) esferas de governo; revoga dispositivos das Leis nos 8.080, de 19 de setembro de 1990, e 8.689, de 27 de julho de 1993; e dá outras providências. Brasília, 2012.

BRASIL. Lei $\mathrm{n}^{\circ} 8.080$, de 19 de setembro de 1990. Dispõe sobre as condições para a promoção, proteção e recuperação da saúde, a organização e o funcionamento dos serviços correspondentes e dá outras providências. Diário Oficial da União, Brasília, DF, 20 set. 1990. p. 18055.

BRASIL. Ministério da Saúde. Financiamento público de saúde / Ministério da Saúde, Organização Pan-Americana da Saúde. - Brasília: Ministério da Saúde, 2013.

BRASIL. Ministério da Saúde. Fundo Nacional de Saúde. Gestão Financeira do Sistema Único de Saúde: manual básico / Ministério da Saúde, Fundo Nacional de Saúde. - $3^{\mathrm{a}}$ Ed. rev. e ampl. - Brasília: Ministério da Saúde, 2003.

BRASIL. Ministério Da Saúde. Portaria n ${ }^{\circ} 204 /$ GM de 29 de janeiro de 2007. Regulamenta o financiamento e a transferência dos recursos federais para as ações e os serviços de saúde, na forma de blocos de financiamento, com o respectivo monitoramento e controle. Brasília, DF. 2007.

BRASIL, Ministério da Saúde. Portaria n ${ }^{\circ} 837$, de 23 de abril de 2009. Altera e acrescenta dispositivos à Portaria $n^{\circ}$ 204/GM, de 29 de janeiro de 2007, para inserir o Bloco de Investimentos na Rede de Serviços de Saúde na composição dos blocos de financiamento relativos à transferência de recursos federais para as ações e os serviços de saúde no âmbito do Sistema Único de Saúde - SUS. Brasília, 2009.

BRASIL, Ministério da Saúde. Portaria interministerial $n^{\circ} 421$, de 3 de março de 2010. Institui o Programa de Educação pelo Trabalho para a Saúde (PET Saúde) e dá outras providências.
Brasília, 2010.

BRASIL, Ministério da Saúde. Programa de Atenção Básica (PAB): Parte Fixa. Brasília: Ministério da Saúde, 2001.

BRASIL, Ministério da Saúde. RENASES - Relação Nacional de Ações e Serviços de Saúde. Brasília, 2012.

BRASIL, Ministério da Saúde. Secretaria de Atenção à Saúde - Departamento de Atenção Básica. Política Nacional de Atenção Básica. Brasília: Ministério da Saúde, 2012.

GERHARDT, T. E.; SILVEIRA, D. T. Métodos de Pesquisa. Universidade Aberta do Brasil - UAB/UFRGS. Curso de Graduação Tecnológica - Planejamento e Gestão para o Desenvolvimento Rural da SEAD/UFRGS. Porto Alegre: Editora da UFRGS, 2009.

PORTALVR - Portal de Transparência. Planejamento Orçamentário. Disponível em: http://www.portalvr.com/transparencia/ $\bmod / \mathrm{planejamento \_ orcamentario/.}$ Acesso em: 27 de agosto de 2017.

SOLHA, R. K. T. Sistema Único de Saúde: componentes, diretrizes e políticas públicas. 1 ed. São Paulo: Érica, 2014. Encontros Regionais sobre Indicadores de Saúde Para Pactuação no Estado do Rio de Janeiro - 2017. Série Histórica dos Indicadores para Pactuação de Metas no Âmbito do Estado do Rio De Janeiro. Governo do Estado do Rio de Janeiro. Obtenção em Secretaria Municipal de Saúde de Volta Redonda - Epidemiologia. Versão: 09.05.17

BRASIL. Fundo Nacional de Saúde. Consulta detalhada bloco Atenção Básica. Disponível em: $\quad$ https://consultafns.saude.gov.br/\#/ detalhada/acao. Acesso em: 25 de Junho de 2017

BRASIL. Fundo Nacional de Saúde. Consulta detalhada bloco Investimento. Disponível em: 
https:/consultafns.saude.gov.br/\#/detalhada/ acao. Acesso em: 25 de Junho de 2017

BRASIL. Fundo Nacional de Saúde. Consulta detalhada bloco Investimento. Disponível em: https://consultafns.saude.gov.br/\#/detalhada/ acao. Acesso em: 25 de Junho de 2017

Secretaria Municipal de Saúde de Volta redonda. Plano Municipal de Saúde 2011/2013. Disponível em: http://www.voltaredonda.rj.gov. $\mathrm{br} / \mathrm{sms} /$ images/relatorios/PlanoMunicipal/ PMS\%20Volta\%20Redonda\%202011-2013. pdf. Acesso em: 15 de Agosto de 2017

Secretaria Municipal de Saúde de Volta redonda. Plano Municipal de Saúde 2014-2017. Disponível em: http://www.voltaredonda.rj.gov. br/sms/images/relatorios/PlanoMunicipal/ PMSVR.pdf. Acesso em: 15 de Agosto de 2017. 